\title{
Delay Line Device
}

National Cancer Institute

\section{Source}

National Cancer Institute. Delay Line Device. NCI Thesaurus. Code C49913.

A communication or electronic circuit designed to delay the transmission of a signal by a set amount. 